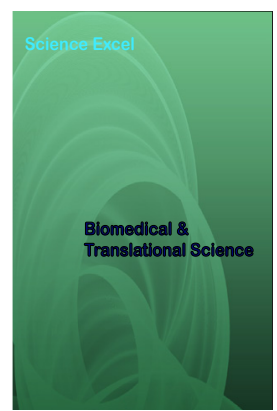

Correspondence

Mouna Lyoubi

Mouna Lyoubi, ENT and Head and Neck Surgery, Hospital August 20, 1953, Ibn Rochd Teaching Hospital, Casablanca,Morocco.

Tel: +212677039404

- Received Date: 04 April 2020

- Accepted Date: 18 June 2020

- Publication Date: 25 June 2020
E-mail:mounalyoubi03@gmail.com

\section{Unusual location of tuberculosis: laryngeal form}

\author{
Mouna Lyoubi, Bushra Abdulhakeem, Hicham Lyoubi, Sanaa mellouk, \\ Youssef Oukessou, Reda Allah Abada, Sami Rouadi, Mohammed Roubal and \\ Mohammed Mahtar
}

ENT and Head and Neck Surgery, Hospital August 20, 1953, Ibn Rochd Teaching Hospital, Casablanca, Morocco.

\begin{abstract}
Introduction:Tuberculosis is a specific infectious disease caused by a mycobacterium: Koch's bacillus. In Morocco, the lung's lesion is the most common site $52 \%$, however lymph node tuberculosis is the most frequent extrapulmonary form. Laryngeal tuberculosis is rare. We present the case of a young man with a laryngeal location.

Presentation of case: A 25-years-old young man, with 10-years history of alcohol and smoking consumption, was presented to our ENT department with a permanent dysphonia and dyspnea on exertion, the patient had fever and weight loss estimated to $15 \mathrm{~kg}$. Clinical examination found a cachectic patient with a $38.5^{\circ} \mathrm{C}$ of temperature, without any palpable cervical mass or lymphadenopathy. A nasofibroscopy was performed showing a right vocal cord's lesion. We performed a cervical CT scan revealing a process of the glottic and supraglottic level. Direct laryngoscopy was performed, objectifying ulcerative mass budding from the right vocal cord. Histopathological examination revealed a tuberculoid granuloma without caseating necrosis. Discussion and conclusion: Laryngeal tuberculosis is a rare and frequently under-recognized disease. It typically affects adults in the fifth decade. Literature reports dysphonia to be the most common symptom, followed by weight loss, cough, dysphagia, and odynophagia. Direct laryngoscopy allows direct visualization of the endolaryngeal lesions and performing multiple biopsies. Histopathological examination may identify granulomatous inflammation, caseating granulomas, and acid fast bacillus. However, the presence of pseudoepitheliomatous hyperplasia, which mimics squamous cell carcinoma, can make the diagnosis difficult. The pharmacologic treatment is the same as for the pulmonary forms, and results are generally excellent. Surgical intervention is reserved for those cases of airway compromise.
\end{abstract}

Copyright

(C) 2020 Science Excel. This is an open access article distributed under the terms of the Creative Commons Attribution 4.0 International license.

\section{Introduction}

Tuberculosis is a specific infectious disease caused by a mycobacterium: Koch's bacillus. It is a public health problem in Morocco. According to the latest WHO updates, the number of tuberculosis cases was 36,000 in 2016, an incidence of 103 new episodes of tuberculosis per 100,000 inhabitants [1]. In Morocco, the lung's lesion is the most common site $52 \%$ [1], however lymph node tuberculosis is the most frequent extrapulmonary form [2], 95\% are located in the cervical region [3]. Laryngeal tuberculosis is rare and in the majority of cases, it's misdiagnosed. The aim of this case report is to describe an unusual location of tuberculosis, emphasizing its pseudo-tumor form.

\section{Case presentation}

AA 25-years-old young man, prisoner that has been released 5 months ago, with 10 -years history of alcohol and smoking consumption and no history of previous pulmonary tuberculosis, was presented to our ENT department with a 7 months' history of permanent dysphonia that was paroxysmal in the beginning, complicated afterwards by dyspnea on exertion, the patient had fever and weight loss estimated to $15 \mathrm{~kg}$.

Clinical examination found a cachectic patient with a $38.5^{\circ} \mathrm{C}$ of temperature, without any palpable cervical mass or lymphadenopathy. A nasofibroscopy was performed showing a right vocal cord's lesion. We performed a cervical CT scan revealing a process of the glottic and supraglottic level measuring $33.6 \mathrm{~mm}$ without cervical lymphadenopathy (Figure 1).

Direct laryngoscopy under sedation was performed, objectifying ulcerative mass budding from the right vocal cord extended upwards towards the ventricular band, posteriorly it infiltrated the interarytenoid region and anteriorly the anterior commissure. Histopathological examination 


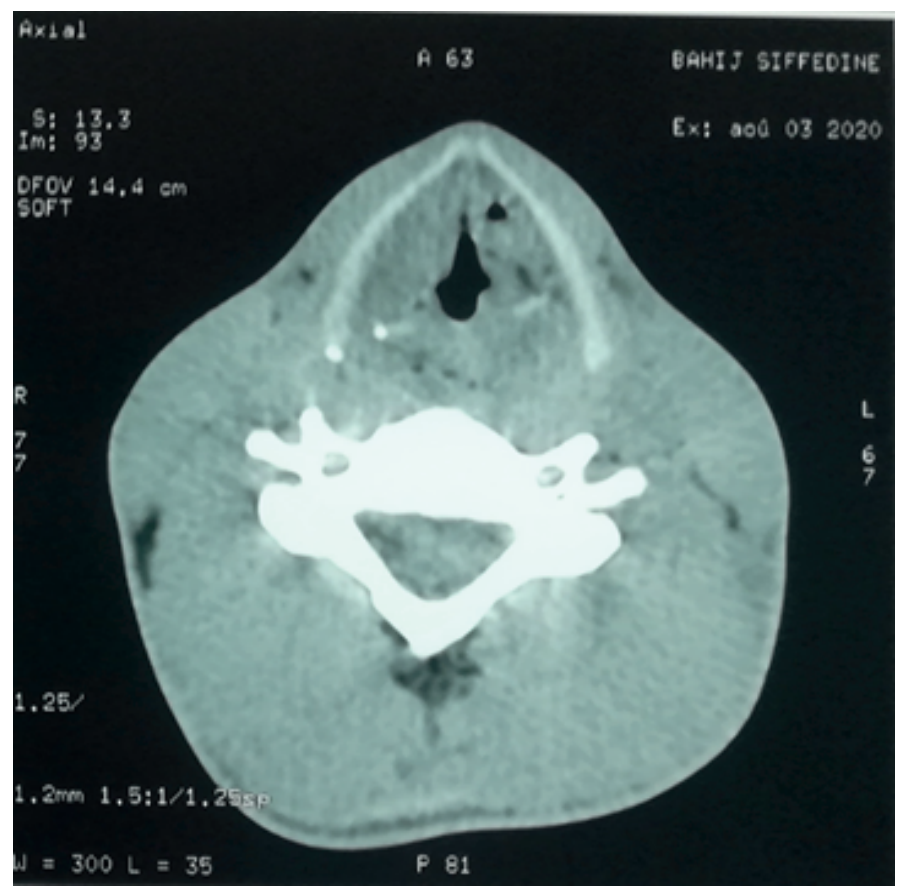

Figure 1: Axial section passing through the glottic plane of the larynx and showing a thickening of the right vocal cord without cartilage lysis.

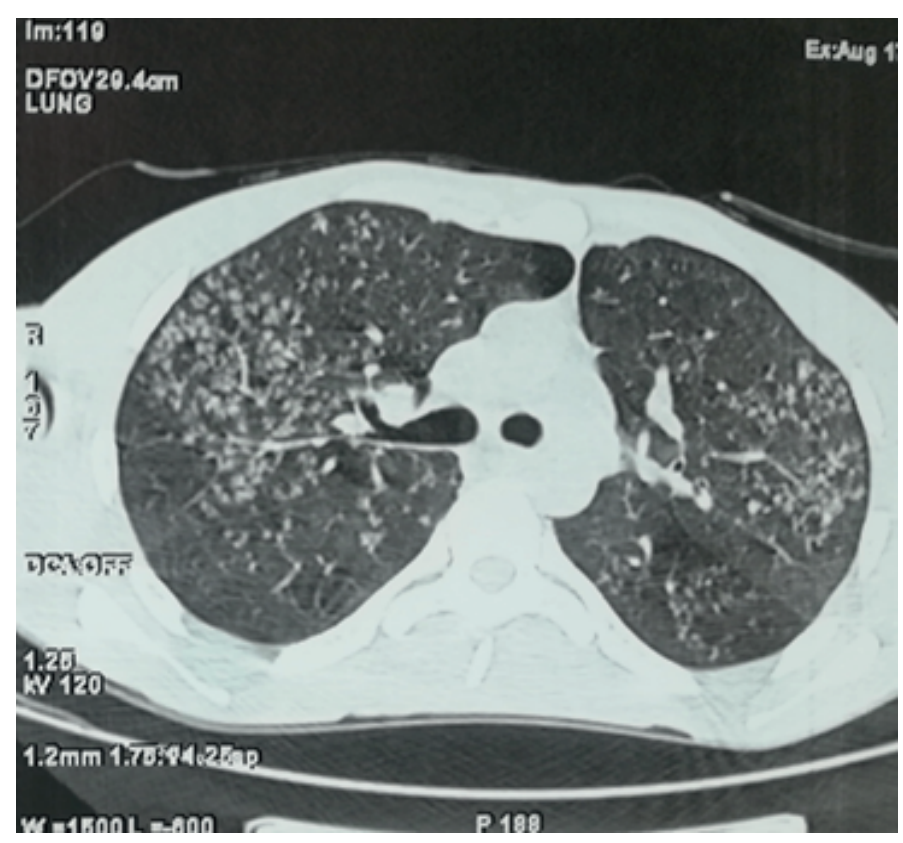

Figure 2: Axial section of the thorax showing an aspect of miliary tuberculosis. revealed a tuberculoid granuloma without caseating necrosis. The assessment was completed by a chest CT scan showing multiple micronodules in both lung fields compatible with pulmonary tuberculosis (Figure 2). The patient was referred to the tuberculosis care center for specific treatment.

\section{Discussion}

Laryngeal tuberculosis is a rare and frequently underrecognized disease. It comprises approximately $1 \%$ of all tuberculosis cases in the United States. [4,5]. It typically affects adults in the fifth decade, and can involve any part of the larynx with a spectrum of presentations, including exophytic, ulcerative, polypoidal and nodular lesions. A review of 127 cases between 1970 and 2012 reported dysphonia to be the most common symptom, followed by weight loss, cough, dysphagia, and odynophagia.

Currently, there are 2 theories that can explain the etiology of laryngeal tuberculosis. The first one is called the bronchogenic theory and states that the larynx is infected by direct spread from the endobronchial tree [6]. This accounts for the majority of cases. The other theory is called the hematogenous theory, and it states that the larynx is infected by hematogenous spread from sites other than the lungs [6]. These patients do not show pulmonary involvement as demonstrated by a normal CT scan.

According to the study of Reis et al, laryngeal tuberculosis patients may present history of previous pulmonary tuberculosis treatment and a long evolution of the disease [7]. Furthermore, the lack of specific symptoms and the insidious nature of laryngeal tuberculosis, together with the difficult to access medical attention and specialized exams [8], may delay diagnosis. However, Wang et al (2007) had found no evidence that delay diagnosis predisposes the patient to larger laryngeal lesions [5].

Direct laryngoscopy allows direct visualization of the endolaryngeal lesions and performing multiple biopsies that should be taken from all suspicious lesions. Histopathological examination may identify granulomatous inflammation, caseating granulomas, and acid fast bacillus. However, the presence of psuedoepitheliomatous hyperplasia, which mimics squamous cell carcinoma, can make the diagnosis difficult; thus, the pathologist should keep in mind that tuberculosis is part of the differential diagnosis. Also, laryngeal tuberculosis and laryngeal squamous cell carcinoma can coexist in the same patient, and laryngoscopy findings are often identical.

There are so many differential diagnosis of laryngeal tuberculosis including neoplasms (mainly squamous cell carcinoma), sarcoidosis, Wegener's granulomatosis, mycotic infections, syphilis, and chronic nonspecific laryngitis $[9,10]$.

The pharmacologic treatment is the same as for the pulmonary forms, and results are generally excellent. Surgical intervention (tracheostomy) is reserved for those cases of airway compromise. After therapy, the patient's symptoms should resolve. However, in those cases of persistent dysphonia, further examinations are required.

\section{Conclusion}

The numerous physicians (internists, general practitioners, otorhinolaryngologists, pathologists, etc.) who deal with the 
various laryngeal symptoms and diseases should be aware of the existence of laryngeal tuberculosis because late diagnosis of this highly contagious disease puts health workers and other patients at risk of occupational and nosocomial infections.

\section{Acknowledgement}

\section{None}

\section{Conflict of Interest}

All authors have no conflict of interest or financial support with this article.

\section{References}

1. https://www.smmg.ma/publications/documents/1-programmenational-de-lutte-contre-la-tuberculose/file.html.

2. Royaume du Maroc Ministère de la Santé Direction de l'Epidémiologie et de lutte contre les maladies. Programme national de lutte anti tuberculeuse - Edition Avril 2011.

3. Ghizlane H, Lamyae A, Hafsa S, et al. Lymph node tuberculosis: epidemiology, diagnostic and therapeutic aspects, about 357 cases. Pan Afr Med J. 2014;19:157.

4. Benwill JL, Sarria JC. Laryngeal tuberculosis in the United States of America: a forgotten disease. Scand J Infect Dis. 2014;46:241-9.

5. Wang CC, Lin CC, Wang CP, et al. Laryngeal tuberculosis: a review of 26 cases. Otolaryngol Head Neck Surg 2007;137:582-8.

6. Ramadan HH, Tarazi AE, Baroudy FM. Laryngeal tuberculosis: Presentation of 16 cases and review of the literature. J Otolaryngo.1993;122:39-41.

7. Reis JGC, Reis CSM, Costa DCSd, et al. Factors Associated with Clinical and Topographical Features of Laryngeal Tuberculosis. PLoS ONE. 2016;11(4):e0153450.

8. Silva L, Damrose E, Bairao F, Nina ML, Junior JC, Costa HO. Infectious granulomatous laryngitis: a retrospective study of 24 cases. Eur Arch Otorhinolaryngology. 2008;265(6):675-80.

9. Couldery AD. Tuberculosis of the upper respiratory tract misdiagnosed as Wegener's granulomatosis--an important distinction. J Laryngol Otol. 1990;104:255-258.

10. Riley EC, Amundson DE: Laryngeal Tuberculosis revisited. Am Fam Physician. 1992;49:759-762. 